\title{
Hamiltonian model and dynamic analyses for a hydro-turbine governing system with fractional item and time-lag
}

\author{
Beibei Xu ${ }^{1}$, Diyi Chen ${ }^{1,2}$, Hao Zhang ${ }^{1}$, Feifei Wang ${ }^{1}$, Xinguang Zhang ${ }^{2}$, Yonghong $\mathrm{Wu}^{2}$ \\ ${ }^{1}$ Institute of Water Resources and Hydropower Research, Northwest A\&F University, Shaanxi \\ Yangling 712100, P. R. China \\ ${ }^{2}$ Department of Mathematics and Statistics, Faculty of Science and Engineering Curtin University, \\ Perth Western Australia 6845, Australia
}

\section{Corresponding author: Diyi Chen}

Mailing Address: Institute of Water Resources and Hydropower Research, Northwest A\&F University, Shaanxi Yangling 712100, China

Telephones: 086-181-6198-0277

E-mail: diyichen@nwsuaf.edu.cn

\begin{abstract}
This paper focus on a Hamiltonian mathematical modeling for a hydro-turbine governing system including fractional item and time-lag. With regards to hydraulic pressure servo system, an universal dynamical model is proposed, taking into account the viscoelastic properties and low-temperature impact toughness of constitutive materials as well as the occurrence of time-lag in the signal transmissions. The Hamiltonian model of the hydro-turbine governing system is presented using the method of orthogonal decomposition. Furthermore, a novel Hamiltonian function that provides more detailed energy information is presented, since the choice of the Hamiltonian function is the key issue by putting the whole dynamical system to the theory framework of the generalized Hamiltonian system. From the numerical experiments based on a real large hydropower station, we prove that the Hamiltonian function can describe the energy variation
\end{abstract}


of the hydro-turbine suitably during operation. Moreover, the effect of the fractional $\alpha$ and the time-lag $\tau$ on the dynamic variables of the hydro-turbine governing system are explored and their change laws identified, respectively. The physical meaning between fractional calculus and time-lag are also discussed in nature. All of the above theories and numerical results are expected to provide a robust background for the safe operation and control of large hydropower stations.

KEY WORDS: Hamiltonian function, Hamiltonian model, hydro-turbine governing system, fractional calculus, time-lag

\section{Introduction}

By 2014, China's total installed capacity of hydropower has passed three hundred million kilowatts, which represents about $25 \%$ of the current installed capacity of hydropower worldwide. Moreover, according to current projections provided by international hydropower industries and referred to the next thirty years, a significant growth in the sector is expected to bring the worldwide installed capacity from ten hundred to twenty hundred million kilowatts. Undoubtedly, the growth of hydropower represents a promising prospect. There are still several outstanding problems in the operation of large hydropower stations [1-3], such as the vibration of hydro-turbine generator units. So far, some large hydropower stations, e.g. Yantan, Liujiaxia, Ertan, Gezhouba, Three Gorges, and Tianhuangping, have already appeared obvious vibration. A recent study reveals that there is a link between the vibration and the operation range of a hydro-turbine-generator unit. The ranges of operation are however inseparably linked to the regulation of the hydro-turbine governing system. Several studies available in the literature focused on the definition of models for the study of ranges of operation and dynamics [4-12]. For example, Nagode et al. [4] proposed a mathematical model of a Francis turbine with a surge tank and suggested a novel method for the control of a hydropower 
plant; Li et al. [5] focused on the modeling of a dynamical hydraulic turbine governing systems with nonelastic water hammer effect.

The transmission of speed signal in the hydraulic pressure servo system requires a not-negligible interval of time which causes the delay of the wicket gates operation with respect to the change of the electricity load of the power grid. Unfortunately, the effect of time-lag is not considered in most available mathematical modeling. Thus, the qualities of complex nonlinear, time-variant and non-minimum phase for a hydro-turbine governing system, together with the time-lag, represent challenging issues for the modeling and control of hydropower systems.

The first studies on fractional calculus date back to 1695 , with the introduction of derivative, integral and eventually differential equations. Nowadays, thanks to the rapid development of applied mathematics and computer science, fractional calculus is widely used in several fields of science and engineering [13-21], e.g. dynamics, image processing, contol engineering etc. When compared with integer-order differential, it shows unique advantages in modeling viscoelastic substance, medical image processing [22], compared with integer-order differential. Due to the obvious viscoelastic properties and low-temperature impact toughness of constitutive material for the hydraulic pressure servo system, integer order calculus is a typical algorithm with limited nature and not suitable to describe it.

The generalized Hamiltonian system is a purely academic offshoot of nonlinear science. Recently, it has been applied to the theory of nonlinear systems [23-26]. Its structure matrix and damping matrix provide more energy information as the theory of the generalized Hamiltonian system develops. Moreover, the Hamiltonian function is a sort of generalized energy which can be treated as the Lyapunov function. It alos represents potentially a novel strategy for exploring dynamic behaviors of complex systems [27-29]. Sgino et al. [27] provided a detailed discussion on 
the use of stochastic Hamiltonians for noncritical string field on the basis of matrix models; Geng et al. [28] proposed a new hierarchy of integrable evolution equations which contains the generalized Harry Dym type equation. Considering the qualities of complex nonlinear, time-variant and non-minimum phase and time-lag for a hydro-turbine governing system, it is surely a novel way to study the dynamic behaviors and the energy flow that by putting the hydro-turbine governing system to the theory framework of the generalized Hamiltonian system.

In light of these considerations, the novelty of the present study can be summarized into 4 steps. First, a new general dynamical model for hydro-turbine governing system with fractional item and time-lag is proposed. This approach is integrated in the frame of generalized Hamiltonian system by utilizing the method of orthogonal decomposition. Second, we present a new Hamiltonian function that provides more detailed energy information from the numerical experiments that from a real large hydropower station. Third, the effect of time-lag on the dynamic variables and its change laws are investigated through numerical applications. Similarly, the fractional order on the influence of the dynamic variables and change laws are also defined. Finally, the essential differences between fractional calculus and time-lag are discussed in detail.

The present paper is organized as follows: Section 2 describes the proposed strategy for dynamical and Hamiltonian modeling of hydro-turbine governing system with fractional item and time-lag. Numerical experiments along with detailed analyses are presented in Section 3, where the essential difference between fractional calculus and time-lag are also discussed. Finally, the conclusions are presented in Section 4.

Table 1 Variables of the hydro-turbine governing system.

\begin{tabular}{cl}
\hline \hline Symbol & \multicolumn{1}{c}{ Quantity } \\
\hline$x_{1}$ & state variable of penstock system, p.u. \\
$x_{2}$ & state variable of penstock system, p.u. \\
$x_{3}$ & state variable of penstock system, p.u.
\end{tabular}




\begin{tabular}{|c|c|}
\hline$T_{0}$ & elastic time constant of the penstock, p.u. \\
\hline$h_{\mathrm{q}}$ & Relative of head loss of the penstock \\
\hline$Z_{0}$ & Relative values of impedance of surges, p.u. \\
\hline$q_{1}$ & relative flow of hydro turbine, p.u. \\
\hline$h_{0}$ & gross head of hydropower station, p.u. \\
\hline$h_{\mathrm{qT}}$ & Relative value of head of diversion tunnel p.u. \\
\hline$f_{\mathrm{T}}$ & coefficient of head loss of diversion tunnel, p.u. \\
\hline$f_{\mathrm{p}}$ & coefficient of head loss of the penstock, p.u. \\
\hline$y_{\mathrm{r}}$ & deviation of incremental guide vane/wicket gate position, p.u. \\
\hline$y$ & the incremental deviation of the guide vane opening, p.u. \\
\hline$q_{\mathrm{nl}}$ & relative flow of the operation of a system with no load, \\
\hline$D_{\mathrm{t}}$ & damping coefficient of synchronous generator, \\
\hline$h_{\mathrm{t}}$ & relative value of hydro-turbine head, p.u. \\
\hline$A_{\mathrm{t}}$ & coefficient scale, p.u. \\
\hline$\omega$ & deviation of relative angular speed, p.u. \\
\hline$k_{\mathrm{d}}$ & differential adjustment coefficient, \\
\hline$k_{\mathrm{p}}$ & proportional adjustment coefficient, \\
\hline$k_{\mathrm{i}}$ & integral adjustment coefficient, \\
\hline$u$ & control signal of PID controller, \\
\hline$r$ & reference input, p.u. \\
\hline$T_{y}$ & major relay connecter response time, $\mathrm{s}$ \\
\hline$P_{\mathrm{m}}$ & output power of hydro-turbine, p.u. \\
\hline$m_{\mathrm{t}}$ & the deviation of the incremental torque, p.u. \\
\hline$H_{1}$ & Hamiltonian function of unit 1, p.u. \\
\hline $\mathrm{H}_{2}$ & Hamiltonian function of unit 1, p.u. \\
\hline$\omega_{\mathrm{B}}$ & rated angular speed of synchronous generator, $\mathrm{rad} / \mathrm{s}$ \\
\hline$\delta$ & rotor angle of synchronous generator, rad \\
\hline$D$ & damping factor of synchronous generator, p.u. \\
\hline$m_{\mathrm{g}}$ & rate torque of synchronous generator, p.u. \\
\hline$E_{\mathrm{q}}{ }^{\prime}$ & internal transient voltage, p.u. \\
\hline$T_{\mathrm{d} 0}$ & transient time constant of axis $d$, p.u. \\
\hline$U_{\mathrm{s}}$ & voltage of infinite power system \\
\hline$E_{\mathrm{f}}$ & output of excitation controller, p.u. \\
\hline
\end{tabular}

\section{Hamiltonian model of a hydro-turbine governing system with fractional item and time-lag}

\subsection{Generalization}

Most large Chinese hydropower stations are characterized by layouts of one unit with signal penstock, consisting of an upper reservoir, a diversion tunnel, a surge tank, a penstock, a hydro-turbine-generator unit and a draft tube. A general layout of such installations is shown in Fig. 
1.

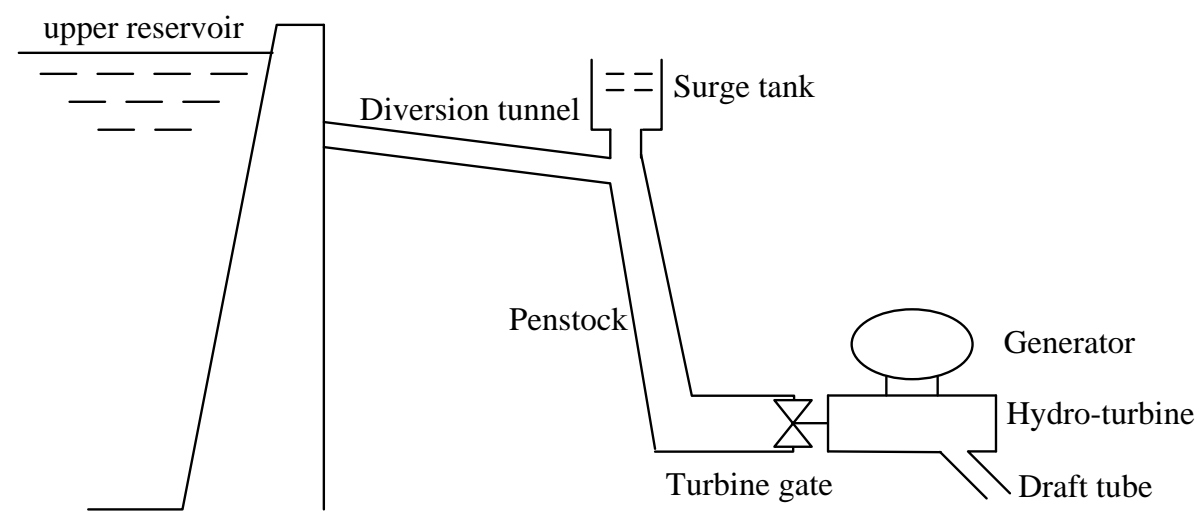

Fig. 1. A general layout of a large hydropower station with a surge tank.

As shown in Fig. 1, the hydro-turbine governing system of this type of large hydropower stations can be divided into two parts. The first includes the hydro-turbine system including the upstream reservoir, the diversion tunnel, the surge tank, the penstock and the hydro-turbine unit. The second consists of the generator system only including the synchronous generator unit.

\subsection{Dynamical modeling of the hydro-turbine system with fractional item and time-lag}

The dynamic behaviors of diversion tunnel are expressed by

$$
\left\{\begin{array}{l}
h_{r}=h_{0}-h_{f T}-h_{q T} \\
h_{f T}=f_{p T} q_{T}^{2} \\
h_{q T}=T_{\omega c} \frac{d q_{T}}{d t}
\end{array},\right.
$$

where $h_{r}$ is the head of the surge tank, $h_{0}$ the static head of the water column, $h_{f T}$ the friction head loss in the diversion tunnel, the $h_{q T}$ the head loss caused by water hammer in the diversion tunnel, $f_{p T}$ the friction factor of the diversion tunnel, $q_{T}$ the water flow in the diversion tunnel, and $T_{\omega c}$ water inertia time. Water inertia time constant $T_{\omega c}[31]$ is given by

$$
T_{\omega}=\frac{Q_{r}}{g H_{r}} L
$$

where $Q_{r}$ is rated discharge, $H_{r}$ rated head, $g$ acceleration of gravity, and $L$ the length of diversion tunnel. With reference to the surge tank, the equations are 


$$
\left\{\begin{array}{l}
h_{r}=\frac{1}{C_{s}} \int q_{s} d t-f_{s} q_{s}^{2} \\
q_{1}=q_{T}-q_{s}
\end{array},\right.
$$

where $q_{s}$ is the water flow in the surge tank, $f_{s}$ the friction factor of the surge tank, and $q_{1}$ the discharge of the hydro-turbine. According to second Newton's law, penstock dynamics are

$$
\left\{\begin{array}{l}
h_{t 1}=h_{r}-h_{f 1}-h_{q 1} \\
h_{f 1}=f_{1} q_{1}^{2} \\
h_{q 1}=Z_{01} q_{1} \tanh \left(T_{01} s\right)
\end{array},\right.
$$

where $h_{t 1}$ is the head of the hydro-turbine, $h_{f 1}$ is the friction head loss in the penstock, the $h_{q 1}$ is the head loss caused by water hammer in the penstock, and $f_{1}$ is the friction factor of the penstock. Considering the elastic water hammer effect in the penstock, the head loss $h_{q 1}$ can be rewritten as [23]

$$
h_{q 1}(s)=Z_{01} \frac{\pi^{2} T_{01} s+T_{01}^{3} s^{3}}{\pi^{2}+4 T_{01}^{2} s^{2}} q_{1}(s)
$$

Eq. (5) can be rewritten as the following state space equations:

$$
\left\{\begin{array}{l}
\dot{x}_{1}=x_{2} \\
\dot{x}_{2}=x_{3} \\
\dot{x}_{3}=-\frac{\pi^{2}}{T_{01}^{2}} x_{2}+\frac{1}{Z_{01} T_{01}^{3}} h_{q 1} . \\
\dot{q}_{1}=-3 \pi^{2} x_{2}+\frac{4}{Z_{01} T_{01}} h_{q 1}
\end{array}\right.
$$

The hydraulic servo system provides forces to regulate the guide vane opening so that the output power of the hydro-turbine and the frequency of the speed of the generator can meet the requirements of a hydropower station. Its operational principle is shown in Fig. 2.

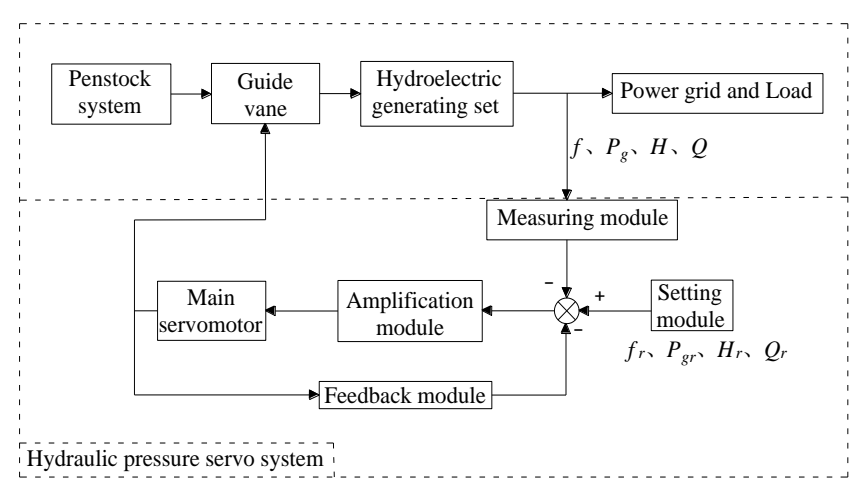


Fig. 2. The operational principle of the hydraulic pressure servo system.

The measuring module, shown in Fig. 2, measures the speed signal of the generator continuously and transmutes it to the voltage signal; the measuring module sends it then to the adder. Once received the signal, the adder sends out a regulate order to the main servomotor, comparing the voltage signal with a given signal from the setting module. Finally, when an imbalance between the torque of the hydro-turbine and the mechanical torque of the synchronous generator occurs, the main servomotor provides a powerful force to promote the change of the opening of the wicket vane. In view of the time cost associated with the above process, the opening of the wicket vane remains unchanged until the main servomotor provides a powerful force. Therefore, this results in an operation process time-lag of hydro-turbine governing system. In this work time-lag is considered in the model of hydraulic pressure servo system, and its equation can be written as

$$
\dot{y}(t)=\frac{1}{T_{y}}\left(u(t)-y(t-\tau)+y_{0}\right) .
$$

Then, considering the viscoelastic properties and low-temperature impact toughness of constitutive materials of the hydraulic pressure servo system, fractional calculus is also introduced into Eq. (7) which can be rewritten as

$$
D^{\alpha} y(t)=\frac{1}{T_{y}}\left(u(t)-y(t-\tau)+y_{0}\right) .
$$

According to the principles of orifice outflow, the head of the hydro-turbine $h_{t 1}$ can be expressed as

$$
h_{t 1}(t)=\frac{y_{r}^{2}}{y(t-\tau)^{2}} q_{1}(t)^{2} .
$$

Similarly, the head loss in the penstock can be rewritten as

$$
h_{q 1}(t)=h_{0}-h_{f T}(t)-h_{q T}(t)-h_{f 1}(t)-h_{t 1}(t)=h_{0}-f_{p T} q_{T}(t)^{2}-T_{\omega c} \dot{q}_{T}(t)-f_{1} q_{1}(t)^{2}-\frac{y_{r}^{2}}{y(t-\tau)^{2}} q_{1}(t)^{2} .
$$

Nowadays, most hydropower stations in China adopt PID controller in the hydraulic pressure servo system. Under this assumption, the output signal $u$ can be written as

$$
u=k_{p}(r-\omega)+k_{i} \int(r-\omega) d t+k_{d}(r-\dot{\omega}) .
$$


Combining Eq. (1) and (11) organically, the mathematical equations regulating the hydro-turbine system with fractional item and time-lag become

$$
\left\{\begin{array}{l}
\dot{x}_{1}(t)=x_{2}(t) \\
\dot{x}_{2}(t)=x_{3}(t) \\
\dot{x}_{3}(t)=-\frac{\pi^{2}}{T_{01}^{2}} x_{2}(t)+\frac{1}{Z_{01} T_{01}^{3}}\left(h_{0}-f_{p T} q_{T}(t)^{2}-T_{\omega c} \dot{q}_{T}(t)-f_{1} q_{1}(t)^{2}-\frac{y_{r}^{2}}{y(t-\tau)^{2}} q_{1}(t)^{2}\right) \\
\dot{q}_{1}(t)=-3 \pi^{2} x_{2}(t)+\frac{4}{Z_{01} T_{01}}\left(h_{0}-f_{p T} q_{T}(t)^{2}-T_{\omega c} \dot{q}_{T}(t)-f_{1} q_{1}(t)^{2}-\frac{y_{r}^{2}}{y(t-\tau)^{2}} q_{1}(t)^{2}\right) \\
\dot{q}_{T}(t)=\frac{1}{T_{\omega c}}\left(h_{0}-h_{r}-f_{p T} q_{T}(t)^{2}\right) \\
\dot{h}_{r}(t)=\frac{1}{C_{s}} q_{s}(t)-2 f_{s} q_{s}(t) \\
D^{\alpha} y(t)=\frac{1}{T_{y}}\left(u(t)-y(t-\tau)+y_{0}\right)
\end{array}\right.
$$

Such system of equations can be expressed as an affine nonlinear mathematical model

$$
\dot{X}(t)=f(x(t), y(t-\tau))+g(x(t)) u(t)
$$

where

$$
\begin{aligned}
& X(t)=\left[\begin{array}{lllllll}
x_{1}(t) & x_{2}(t) & x_{3}(t) & q_{1}(t) & q_{T}(t) & h_{r}(t) & y(t)
\end{array}\right], \quad g(x(t))=\left[\begin{array}{lllllll}
0 & 0 & 0 & 0 & 0 & 0 & \frac{1}{T_{y}}
\end{array}\right], \text { and } \\
& f_{1}(x(t))=\left[\begin{array}{l}
x_{2}(t) \\
x_{3}(t) \\
-\frac{\pi^{2}}{T_{01}^{2}} x_{2}(t)+\frac{1}{Z_{01} T_{01}^{3}}\left(h_{0}-f_{p T} q_{T}(t)^{2}-T_{\omega c} \dot{q}_{T}(t)-f_{1} q_{1}(t)^{2}-\frac{y_{r}^{2}}{y(t-\tau)^{2}} q_{1}(t)^{2}\right) \\
-3 \pi^{2} x_{2}(t)+\frac{4}{Z_{01} T_{01}}\left(h_{0}-f_{p T} q_{T}(t)^{2}-T_{\omega c} \dot{q}_{T}(t)-f_{1} q_{1}(t)^{2}-\frac{y_{r}^{2}}{y(t-\tau)^{2}} q_{1}(t)^{2}\right) \\
\frac{1}{T_{\omega c}}\left(h_{0}-h_{r}-f_{p T} q_{T}(t)^{2}\right) \\
\frac{1}{C_{s}} q_{s}(t)-2 f_{s} q_{s}(t) \\
\frac{1}{T_{y}}\left(y_{0}-y(t-\tau)\right)
\end{array}\right] .
\end{aligned}
$$

The frame diagram of transfer functions of the hydro-turbine governing system with fractional item and time-lag is shown in Fig. 3. 


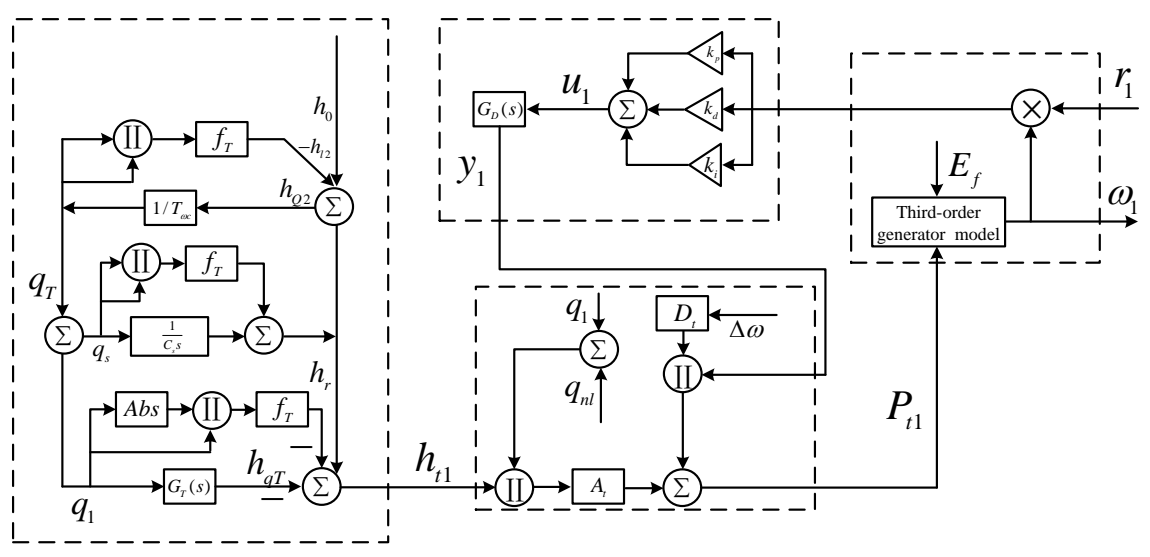

Fig. 3. The transfer functions of the hydro-turbine governing system with fractional item and time-lag

\subsection{Hamiltonian modeling of the hydro-turbine system with fractional item and time-lag}

Assuming that $H_{1}(t)$ is the Hamiltonian function of the hydro-turbine system, its output can be expressed as

$$
H_{\text {output }}(t)=g(X)^{T} \frac{\partial H_{1}(t)}{\partial X}
$$

The relationship between the output power $P_{m}(t)$ of the hydro-turbine and the torque of the generator $m_{t}(t)$ is

$$
P_{m}(t)=m_{t}(t) \omega(t),
$$

where $\omega(t)$ is the generator speed. Hence, from Eqs. (14) and (15), the Hamiltonian function $H_{1}(t)$ can be rewritten as

$$
H_{1}(t)=\int g(X) H_{\text {ouput }}(t)=\int g(X) P_{m}(t)=\int g(X) m_{t}(t) \omega(t) .
$$

The mathematical equation of the output power for a hydro-turbine, proposed by IEEE Working Group in 1993, is often used in the mathematical modeling of a hydro-turbine system [31] in the form shown as

$$
P_{m-I E E E}(t)=A_{t} h_{t}(t)\left(q_{1}(t)-q_{n l}\right)-D_{t} y(t) \omega(t) .
$$

To respect the condition $P_{m-I E E E}(t)>0$, where $P_{m-I E E E}(t)$ is the output power of the hydro-turbine, the following condition must be satisfied 


$$
q_{1}(t)-q_{n l}>\frac{D_{t} y(t-\tau) \omega(t)}{A_{t} h_{t}(t)}>0
$$

Eq. (17) is just a general formula that does not take into account the characteristics of the output power for a selected hydro-turbine in transient process, since the organization structures (the mounting height of the guide vane, the discharge angle of the guide vane etc.) of different types of hydro-turbines are largely different. To overcome this limitation, in this study, the output power dynamics derived from internal characteristics method is represented as

$$
\left\{\begin{array}{l}
h_{t 1}(t)=\frac{\omega(t)}{g}\left[\left(\frac{\cot \gamma}{2 \pi b_{0}}+r \frac{\cot \beta}{F}\right) q_{1}(t)-\omega(t) r^{2}\right] \\
m_{t 1}(t)=\rho\left[\left(\frac{\cot \gamma}{2 \pi b_{0}}+r \frac{\cot \beta}{F}\right) q_{1}(t)-\omega(t) r^{2}\right]
\end{array},\right.
$$

where $\gamma$ is the discharge angle of the guide vane, $\beta$ the discharge angle of the middle area of the runner, $b_{0}$ the mounting height of the guide vane, $r$ the radius of the middle area of the runner, and $F$ the area of the exit of the runner. On the basis of Eqs. (16) and (18), the Hamiltonian function can be rewritten as

$$
H_{1}(t)=\rho T_{y} q_{1}(t) y(t-\tau) \omega(t)\left[\frac{r}{F} \cot \beta+\frac{1}{2 b_{0} \pi} \cot \gamma-r^{2} \frac{\omega(t)}{q_{1}(t)}\right] .
$$

Assuming that $H_{1}(t)$ is a positive definite function, the condition in Eq. (20) is obtained as

$$
r^{2} \frac{\omega(t)}{q_{1}(t)}-\frac{r}{F} \cot \beta-\frac{1}{2 b_{0} \pi} \cot \gamma>0
$$

Utilizing Eq. (19), Eq. (13) can be transformed into the Hamiltonian mathematical model [32], which is

$$
\dot{X}(t)=\left[J_{1}(t, x(t), y(t-\tau))+P_{1}(t, x(t), y(t-\tau))\right] \frac{\partial H_{1}(x(t), y(t-\tau))}{\partial x(t)}+g(x(t)) u(t),
$$

where

$$
J_{1}(t, x(t), y(t-\tau))=\left[\begin{array}{ccccccc}
0 & j_{12} & j_{13} & j_{14} & j_{15} & j_{16} & j_{17} \\
-j_{12} & 0 & j_{23} & j_{24} & j_{25} & j_{26} & j_{27} \\
-j_{13} & -j_{23} & 0 & j_{34} & j_{35} & j_{36} & j_{37} \\
-j_{14} & -j_{24} & -j_{34} & 0 & j_{45} & j_{46} & j_{47} \\
-j_{15} & -j_{25} & -j_{35} & -j_{45} & 0 & j_{56} & j_{57} \\
-j_{16} & -j_{26} & -j_{36} & -j_{46} & -j_{56} & 0 & j_{67} \\
-j_{17} & -j_{27} & -j_{37} & -j_{47} & -j_{57} & -j_{67} & 0
\end{array}\right], \quad P_{1}(t, x(t), y(t-\tau))=\left[\begin{array}{ccccccc}
p & 0 & 0 & 0 & 0 & 0 & 0 \\
0 & p & 0 & 0 & 0 & 0 & 0 \\
0 & 0 & p & 0 & 0 & 0 & 0 \\
0 & 0 & 0 & p & 0 & 0 & 0 \\
0 & 0 & 0 & 0 & p & 0 & 0 \\
0 & 0 & 0 & 0 & 0 & p & 0 \\
0 & 0 & 0 & 0 & 0 & 0 & p
\end{array}\right] .
$$


The variable $p$ appearing in symmetric matrix $P_{i}^{(1)}(x)$ can be expressed as

$$
p=\frac{<f(x), \nabla H>}{\|\nabla H\|^{2}}=\frac{1}{\|\nabla H\|^{2}}\left\{f_{1} \nabla H_{x 1}+f_{2} \nabla H_{x 2}+f_{3} \nabla H_{x 3}+f_{4} \nabla H_{x 4}+f_{5} \nabla H_{x 5}+f_{6} \nabla H_{x 6}+f_{7} \nabla H_{x 7}\right\}=r p+s p .
$$

In light of this, the symmetric matrix $P_{i}^{(1)}(x)$ can be further written as

$$
P_{1}(x(t), y(t-\tau))=S_{1}(x(t), y(t-\tau))-R_{1}(x(t), y(t-\tau)),
$$

where

$$
R_{1}(x, y(t-\tau))=\left[\begin{array}{lllllll}
r p & 0 & 0 & 0 & 0 & 0 & 0 \\
0 & r p & 0 & 0 & 0 & 0 & 0 \\
0 & 0 & r p & 0 & 0 & 0 & 0 \\
0 & 0 & 0 & r p & 0 & 0 & 0 \\
0 & 0 & 0 & 0 & r p & 0 & 0 \\
0 & 0 & 0 & 0 & 0 & r p & 0 \\
0 & 0 & 0 & 0 & 0 & 0 & r p
\end{array}\right], \quad S_{1}(x(t), y(t-\tau))=\left[\begin{array}{lllllll}
s p & 0 & 0 & 0 & 0 & 0 & 0 \\
0 & s p & 0 & 0 & 0 & 0 & 0 \\
0 & 0 & s p & 0 & 0 & 0 & 0 \\
0 & 0 & 0 & s p & 0 & 0 & 0 \\
0 & 0 & 0 & 0 & s p & 0 & 0 \\
0 & 0 & 0 & 0 & 0 & s p & 0 \\
0 & 0 & 0 & 0 & 0 & 0 & s p
\end{array}\right] .
$$

Therefore, the Hamiltonian model of the hydro-turbine governing system with fractional item and time-lag can be described as

$$
D^{\alpha_{i}} X(t, y(t-\tau))=\left[J_{1}(x(t), y(t-\tau))+S_{1}(t, y(t-\tau))-R_{1}(t, y(t-\tau))\right] \frac{\partial H_{1}(t, y(t-\tau))}{\partial x(t)}+g(t) u(t) .
$$

Finally, according to Wang et al. [32], Eq. (24) can be further rewritten as

$$
\dot{X}(t)=\left[J^{\prime}(x(t), y(t-\tau))-R^{\prime}(x(t), y(t-\tau))\right] \frac{\partial H_{1}(x(t), y(t-\tau))}{\partial x(t)}+g(x(t)) u^{\prime} .
$$

\subsection{The Hamiltonian model of the generator system}

In this paper, a third synchronous generator model is used, which expressed by the system of equations

$$
\left\{\begin{array}{l}
\dot{\delta}=\omega_{B}(\omega-1) \\
\dot{\omega}=\frac{1}{T_{j}}\left(P_{m}-m_{g}-D(\omega-1)\right) \\
\dot{E}_{q}^{\prime}=-\frac{\omega_{B}}{T_{d 0^{\prime}}} \frac{X_{d \Sigma}}{X_{d \Sigma^{\prime}}} E_{q}^{\prime}+\frac{\omega_{B}}{T_{d 0^{\prime}}} \frac{X_{d \Sigma}-X_{d \Sigma^{\prime}}}{X_{d \Sigma^{\prime}}} U_{s} \cos \delta+\frac{\omega_{B}}{T_{d 0^{\prime}}} E_{f}
\end{array} .\right.
$$

The Hamiltonian mathematical model of the generator system is [33] 


$$
\left[\begin{array}{c}
\dot{\delta} \\
\dot{\omega} \\
\dot{E}_{q}
\end{array}\right]=\left[\begin{array}{ccc}
0 & C_{1} & 0 \\
-C_{1} & -C_{D} & 0 \\
0 & 0 & -C_{G}
\end{array}\right]\left[\begin{array}{c}
\frac{\partial H_{2}}{\partial \delta} \\
\frac{\partial H_{2}}{\partial \omega} \\
\frac{\partial H_{2}}{\partial E_{q}}
\end{array}\right]+\left[\begin{array}{cc}
0 & 0 \\
A & 0 \\
0 & \frac{\omega_{B}}{T_{d 0}^{\prime}}
\end{array}\right]\left[\begin{array}{c}
m_{t} \\
E_{f}
\end{array}\right] .
$$

Its Hamiltonian function is

$$
H_{2}(t)=\frac{T_{j}}{2}(\omega(t)-1)^{2}+\frac{U_{s}^{2}}{2} \frac{X_{q \Sigma}-X_{d \Sigma}}{X_{q \Sigma} X_{d \Sigma}} \cos ^{2} \delta(t)+\frac{U_{s}^{2}}{2 X_{q \Sigma}}+\frac{1}{2 X_{d \Sigma} X_{d \Sigma} X_{f}}\left(X_{a d} U_{s} \cos \delta(t)-X_{d \Sigma} \frac{X_{f}}{X_{a d}} E_{q}^{\prime}(t)\right)^{2} .
$$

Specific parameters are presented in Zeng et al [33].

\subsection{The Hamiltonian model of the whole system}

Set $D^{\alpha_{i}} X(t)=\left[\dot{x}_{1}(t) \dot{x}_{2}(t) \dot{x}_{3}(t) \dot{q}_{1}(t) \dot{q}_{T}(t) \dot{h}_{r}(t) D^{\alpha} y(t) \dot{\delta}(t) \dot{\omega}(t) \dot{E}_{q}^{\prime}(t)\right]^{T}, \quad H(t)=H_{1}(t)+H_{2}(t)$. From Eqs. (25) and (27), the Hamiltonian mathematical model of the hydro-turbine governing system with fractional item and time-lag is

$$
D^{\alpha_{i}} X(t)=[J(x(t), y(t-\tau))-R(x(t), y(t-\tau))] \frac{\partial H(x(t), y(t-\tau))}{\partial x(t)}+g(x(t)) u(t),
$$

where

$$
\begin{aligned}
H(t)= & \frac{T_{j}}{2}(\omega(t)-1)^{2}+\frac{U_{s}^{2}}{2} \frac{X_{q \Sigma}-X_{d \Sigma}}{X_{q \Sigma} X_{d \Sigma}} \cos ^{2} \delta(t)+\frac{1}{2 X_{d \Sigma} X_{d \Sigma} X_{f}}\left(X_{a d} U_{s} \cos \delta(t)-X_{d \Sigma} \frac{X_{f}}{X_{a d}} E_{q}^{\prime}(t)\right)^{2} \\
& +\rho T_{y} q_{1}(t) y(t-\tau) \omega(t)\left[\frac{r}{F} \cot \beta+\frac{1}{2 b_{0} \pi} \cot \gamma-r^{2} \frac{\omega(t)}{q_{1}(t)}\right]+\frac{U_{s}^{2}}{2 X_{q \Sigma}}
\end{aligned} .
$$

\section{Numerical experiments}

In order to investigate the performances of the proposed model, numerical examples have been implemented. Basic data were elected from an existent large hydropower station and the model applied to the hydro-turbine governing system with a penstock [23]. The parameters adopted for the hydropower station are

Hydro-turbine type HL99.5-LJ-344.2, nominal head $H_{r}=312 \mathrm{~m}$, nominal flow $Q_{r}=53.5 \mathrm{~m} / \mathrm{s}$, installed capacity $N_{r}=150 \mathrm{MW}$, and nominal speed $n_{r}=333.3 \mathrm{r} / \mathrm{min}$. Other parameters are presented in Table 1 .

\subsection{Numerical experiments for the Hamiltonian function}


As discussed in Section 2, the Hamiltonian function defines the structure matrix and the damping matrix of the whole Hamiltonian system. Therefore, the choice of the Hamiltonian function becomes the key issue by putting the hydro-turbine governing system to the theory framework of the generalized Hamiltonian system. The Hamiltonian function of the whole system in this paper includes two parts which are the Hamiltonian function of the hydro-turbine system and the determined Hamiltonian function of the generator system. To select a proper Hamiltonian function of the hydro-turbine system, which are derived from Eq. (17) and Eq. (18) respectively, are compared in this subsection in order to identify the most valid.

According to Eq. (17), the Hamiltonian function can be rewritten as

$$
H_{\text {IEEE }}=\frac{1}{2} D_{t} T_{y} \omega(t) y(t-\tau)^{2}+\frac{\omega(t)}{y(t-\tau)} A_{t} T_{y} q_{1}(t)^{2} y_{r}^{2}\left(q_{1}(t)-q_{n l}\right) .
$$

Numerical results are computed for the following assumptions.

Assumption 1: The discharge angle of the guide vane remains unchanged in the transient process.

Assumption 2: The discharge angle of the middle area of the runner also remains unchanged in the transient process.

Fig. 4 shows the numerical results for $\mathrm{t}=0.3$ second.
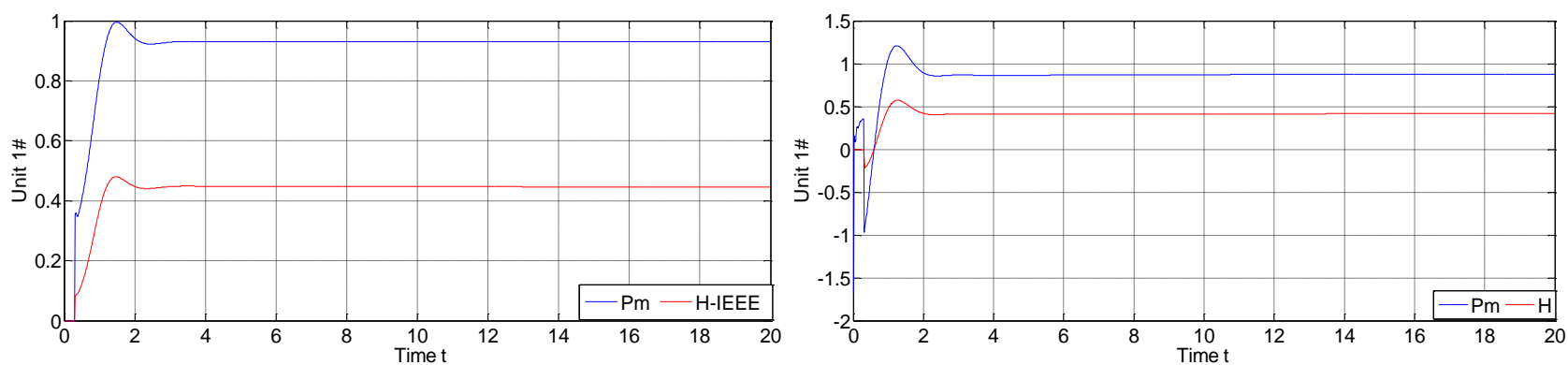

(a) The Hamiltonian function $H_{\text {IEEE }}$ derived from Eq. (17) and the output power $\mathrm{P}_{\text {m-IEEE. }}(b)$ The Hamiltonian function $H$ derived from Eq. (18) and the output power $\mathrm{P}_{\mathrm{m}}$.

Fig. 4. The dynamic behaviors of the Hamiltonian function and the output power with $\tau=0.3$ second. 
As shown in Fig. 4(a) or Fig. 4(b), the output power function has the same trends of the Hamiltonian function. The values of the output power $P_{m}$ and the Hamiltonian function $H_{1}$ grow significantly when the time $t$ increases from 0 to about 1.5 seconds. When time $t$ is over 1.5 second, their values synchronously decrease reaching stability. This trend demonstrate that the Hamiltonian function can adequately describe the dynamic behavior of the hydro-turbine in the transient process.

The obvious dissimilarities are highlighted from the comparison of Fig. 4(a) with Fig. 4(b). First, the output power and Hamiltonian function in Fig. 4(b) appear a palpable reverse regulation phenomenon at about 0.31 second, which is more close to practical engineering. Second, the values of their overshoot in Fig. 4(b) are much larger than that of Fig. 4(a). Third, the stable values of the output power are very different when the system enters into stable operation. The value of the output power derived from Eq. (17) is about 0.93, while the value derived from Eq. (18) becomes about 0.875. This result is due to the two assumptions initially introduced. More specifically, the discharge angle of the guide vane and the discharge angle of the middle area of the runner always remain unchanged with the increase of the discharge $q_{1}$, which causes the increase of the friction head loss directly.

\subsection{The validity of the fractional model with time-lag}

In 2014, Zeng et al [34] proposed a differential algebric model for the BuLuge hydropower station, which is named Zeng's model for convenience in the following. To prove the validity and advantages of the fractional model with time-lag, a detail comparison of the dynamic behaviors of the hydro-turbine discharge $q_{1}$, the head loss $h_{q 1}$, the generator speed $\omega$ and the guide vane opening $y$ for the fractional model with time-lag and Zeng's model are presented (Fig. 5). 


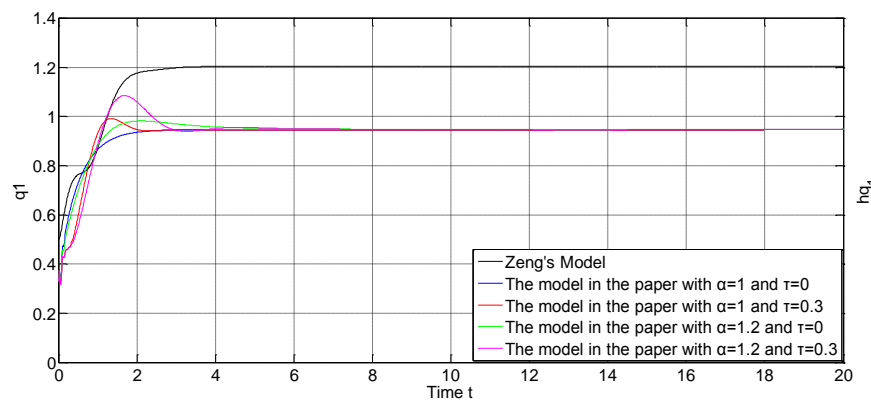

(a) The hydro-turbine discharge $q_{1}$

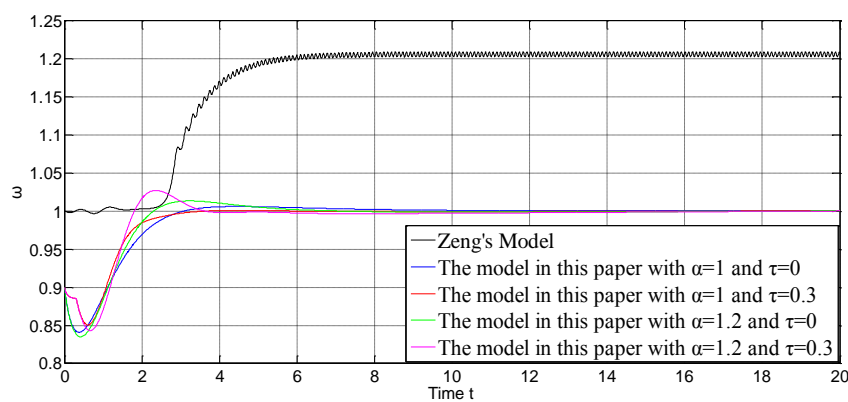

(c) The generator speed $\omega$

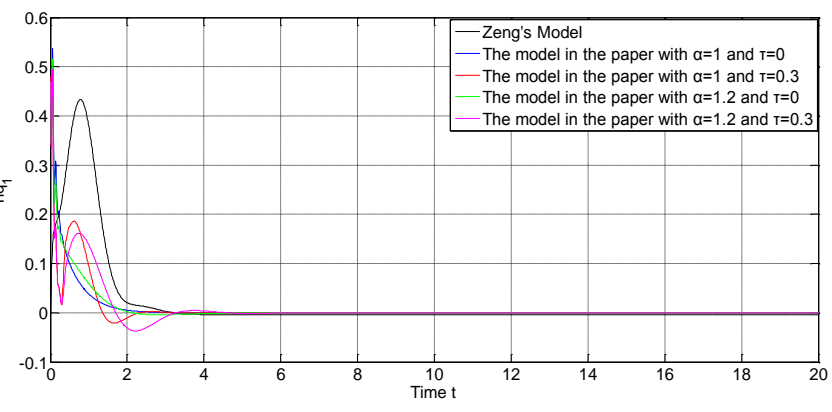

(b) The head loss $h_{q 1}$

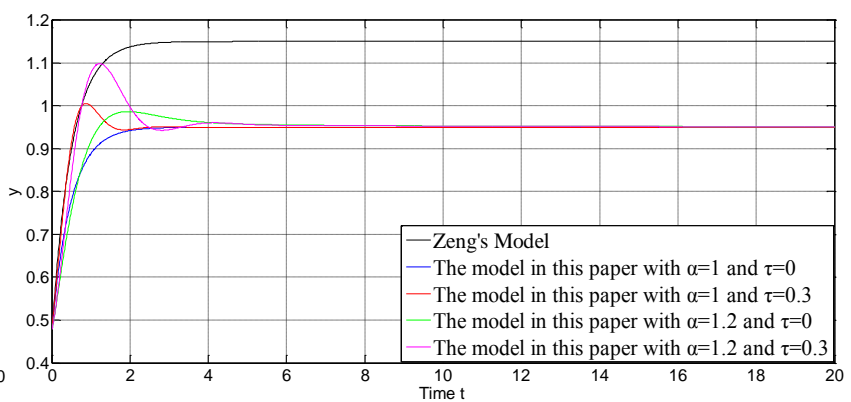

(d) The guide vane opening $y$

Fig. 5. The dynamic behaviors of the hydro-turbine discharge $q_{1}$, the head loss $h_{q 1}$, the generator speed $\omega$, and the guide vane opening $y$ from the different models of the hydro-turbine governing system.

As shown from the graph, compared with the responses of Zeng's model, the dynamic behaviors of the four variables from the integer order model $(\alpha=1, \tau=0)$ decrease obviously and show reverse regulation. The main difference between Zeng's model and the integer order model is that Zeng's model only addresses the elastic water column effect in the mathematical modeling, without considering the effect of surge tank. Nevertheless, the effect of surge tank on the dynamic behavior of the hydro-turbine governing system is apparent. The fractional order $\alpha$ affects the variation of the variables in the transient process, although it does not affect the stable value of the variables. Comparing Zeng's model and the integer order model ( $\alpha=1, \tau=0)$ with the integer order model with time-lag $(\alpha=1, \tau \neq 0)$ and the fractional model with time-lag $(\alpha \neq 1, \tau \neq 0)$, the similarity which is the time-lag $\tau$ has no impact on the stable values of the whole system in the 
stable operation. The difference is that the responses of the variables from the models with time-lag show palpable overshoot signs in the transient process. From the above analysis, the integer order model $(\alpha=1, \tau=0)$ is more exact and reliable in simulating the dynamic behaviors of the hydro-turbine governing system. Moreover, the fractional model with time-lag $(\alpha \neq 1, \tau \neq 0)$ provides more information for the transient process of the hydro-turbine governing system.

\subsection{The influence of time-lag $\tau$ on the dynamic behavior of the hydro-turbine}

To investigate the influence of time-lag $\tau$ on the hydro-turbine dynamics, time-lag $\tau$ is assumes to change from 0.03 second to 0.75 seconds with the increment of 0.03 seconds. The numerical results representing the main dynamic variables of the governing system in function of $\tau$ are shown in Fig. 6. The value of the fractional order $q$ results 1 when the time-lag $\tau$ is being varied.

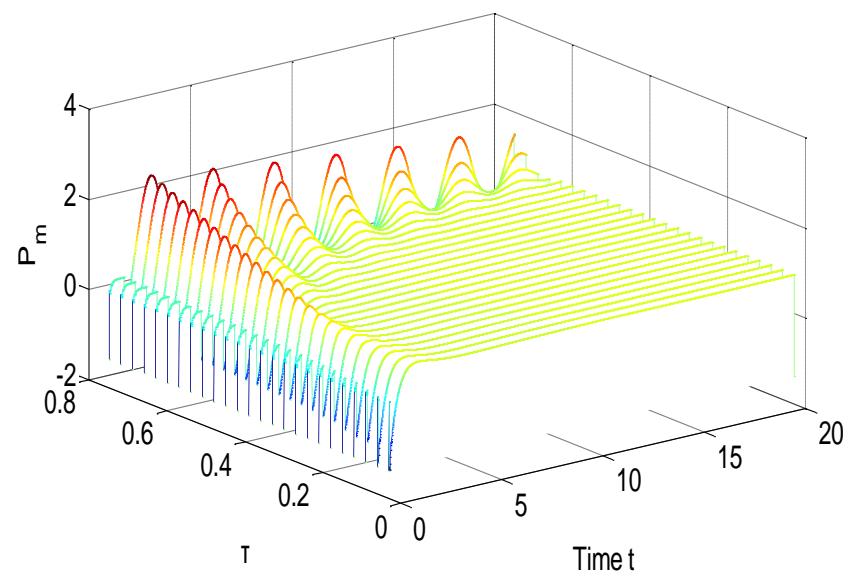

(a) The output power $P_{\mathrm{m}}$

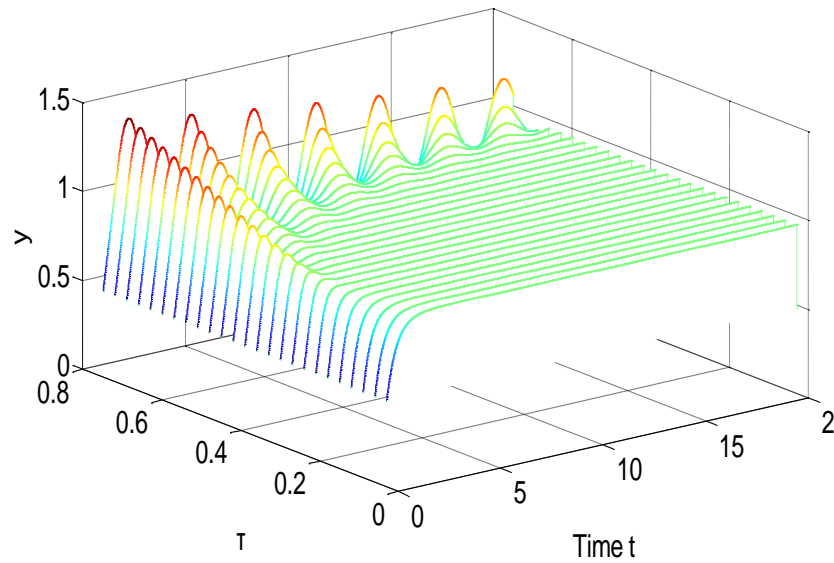

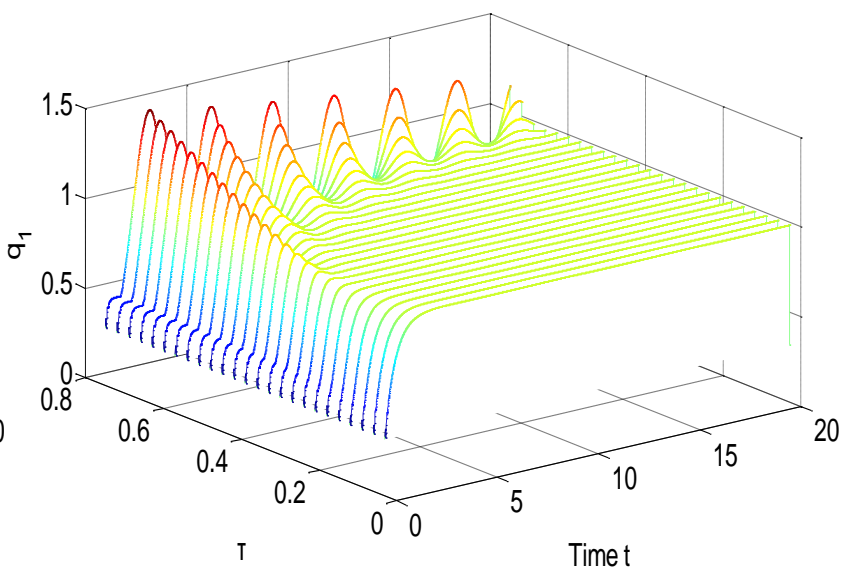

(b) The hydro-turbine discharge $q_{1}$

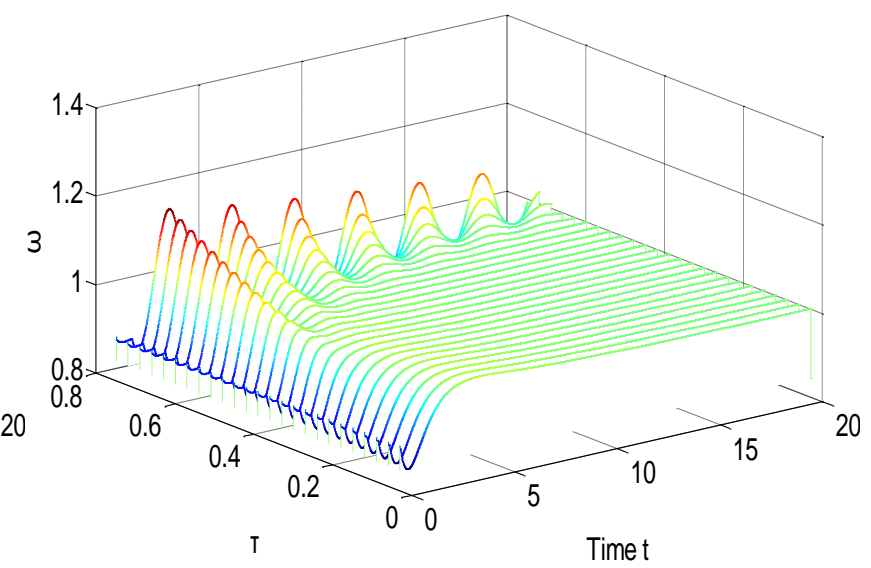



(c) The guide vane opening $y$
(d) The generator speed $\omega$

Fig. 6. The dynamic behavior of the main four variables (the output power $\mathrm{P}_{\mathrm{m}}$, the hydro-turbine discharge $\mathrm{q}_{1}$, the guide vane opening $y$, the generator speed $\omega$ ) of hydro-turbine governing system when $\tau$ changes from 0.03 second to 0.75 second with the increment of 0.03 .

As shown in Fig. 6, the overshoot of the output power, the discharge of the hydro-turbine and the guide vane opening increase along with $\tau$. In contrast with the above three variables, the overshoot of the generator speed decreases gradually. Moreover, a critical value $\tau_{k}$ can be identified, so that when $\tau>\tau_{k}$, the stability of the whole system results influenced by the time-lag. Such trend is captured by the projection of the dynamic variables into the YZ plant plotted in Fig. 7.

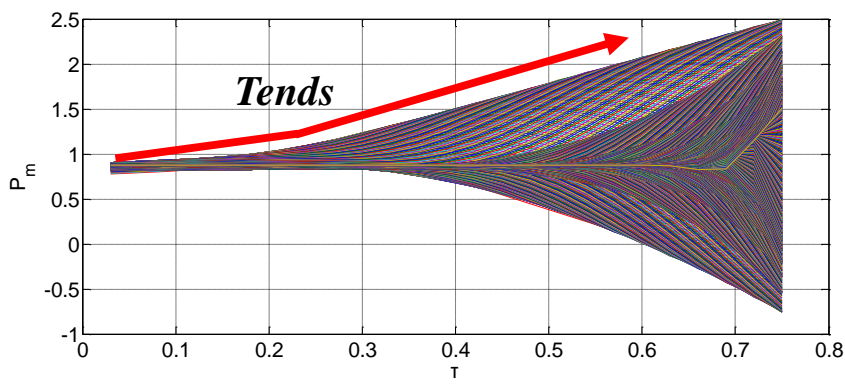

(a) The output power $P_{\mathrm{m}}, 0.03<\tau<0.75$

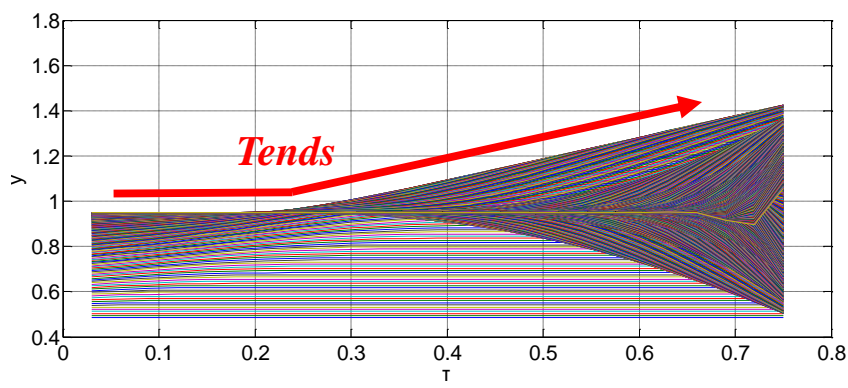

(c) The guide vane opening $y, 0.03<\tau<0.75$

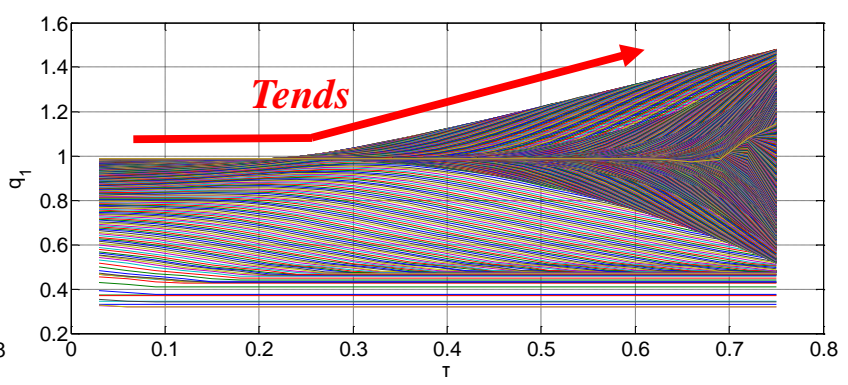

(b) The hydro-turbine discharge $q_{1}, 0.03<\tau<0.75$

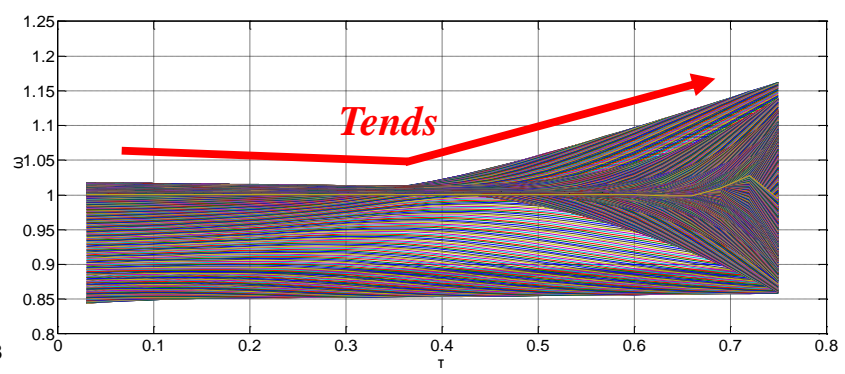

(d) The generator speed $\omega, 0.03<\tau<0.75$

Fig. 7. The change laws of the overshoot of the above main variables (the output power $\mathrm{P}_{\mathrm{m}}$, the hydro-turbine discharge $\mathrm{q}_{1}$, the guide vane opening $y$, the generator speed $\omega$ ) when $\tau$ changes from 0.03 second to 0.75 second with incremental steps of 0.03 .

The time-lag $\tau$ affects the overshoots of the above four variables (the output power $\mathrm{P}_{\mathrm{m}}$, the hydro-turbine discharge $q_{1}$, the guide vane opening $y$, the generator speed $\omega$ ), and the effect laws 
are presented in Tab. 2. It also affects the stability of the four variables as represented by the green lines: the change laws of the stable ranges of the four variables are shown in Table 3.

Table 2 The change laws of the overshoot for the four variables (the output power $P_{\mathrm{m}}$, the hydro-turbine discharge $q_{1}$, the guide vane opening $y$, the generator speed $\omega$ ) of the hydro-turbine governing system with fractional item and time-lag.

\begin{tabular}{ccccc}
\hline Variable & Ranges & Laws & Ranges & Laws \\
\hline$P_{m}$ & $(0.03,0.20)$ & $P_{m}=0.93$ & $(0.20,0.75)$ & $P_{m}=2.81(\tau-0.20)+0.93$ \\
$q_{1}$ & $(0.03,0.24)$ & $q_{1}=0.9866$ & $(0.24,075)$ & $q_{1}=0.962(\tau-0.24)+0.9866$ \\
$y$ & $(0.03,0.21)$ & $y=0.95$ & $(0.21,0.75)$ & $y=0.88(\tau-0.21)+0.95$ \\
$\omega$ & $(0.03,0.35)$ & $\omega=1.02$ & $(0.35,0.75)$ & $\omega=0.355(\tau-0.35)+1.02$ \\
\hline
\end{tabular}

Table 3 The change laws of the four variables (the output power $P_{\mathrm{m}}$, the hydro-turbine discharge $q_{1}$, the guide vane opening $y$, the generator speed $\omega$ ) for the hydro-turbine governing system with fractional item and time-lag.

\begin{tabular}{ccccccc}
\hline Variable & Ranges & Laws & Ranges & Laws & Ranges & Laws \\
\hline$P_{m}$ & $(0.03,0.69)$ & $P_{m}=0.87$ & $(0.69,0.75)$ & $P_{m}=10.65(\tau-0.69)+0.87$ & & \\
$q_{1}$ & $(0.03,0.69)$ & $q_{1}=0.985$ & $(0.69,0.75)$ & $q_{1}=2.67(\tau-0.69)+0.985$ & & \\
$y$ & $(0.03,0.66)$ & $y=0.95$ & $(0.66,0.72)$ & $y=-0.88(\tau-0.66)+0.95$ & $(0.72,0.75)$ & $y=5.29(\tau-0.72)+0.8972$ \\
$\omega$ & $(0.03,0.66)$ & $\omega=1.00$ & $(0.66,0.72)$ & $\omega=0.467(\tau-0.66)+1.00$ & $(0.72,0.75)$ & $\omega=-1.12(\tau-0.72)+1.028$ \\
\hline
\end{tabular}

\subsection{The effect of fractional $\alpha$ on the dynamic behaviors of the hydro-turbine}

In this subsection, the effect of the fractional $\alpha$ on the output power $\mathrm{P}_{\mathrm{m}}$, the hydro-turbine discharge $q_{1}$, the guide vane opening $y$ and the generator speed $\omega$ are explored, respectively. Let the fractional $\alpha$ increase from 0.4 second to 2 second with the incremental steps of 0.08 seconds. The numerical results of the four dynamic variables are shown in Fig. 8. In addition, the value of the time-lag $\tau$ is zero when the fractional order $\alpha$ is being varied. 


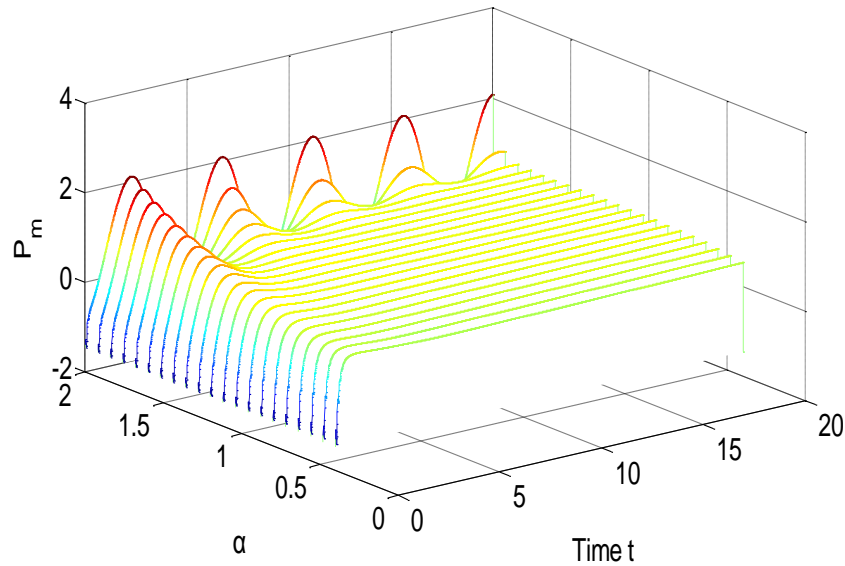

(a) The output power $P_{\mathrm{m}}$

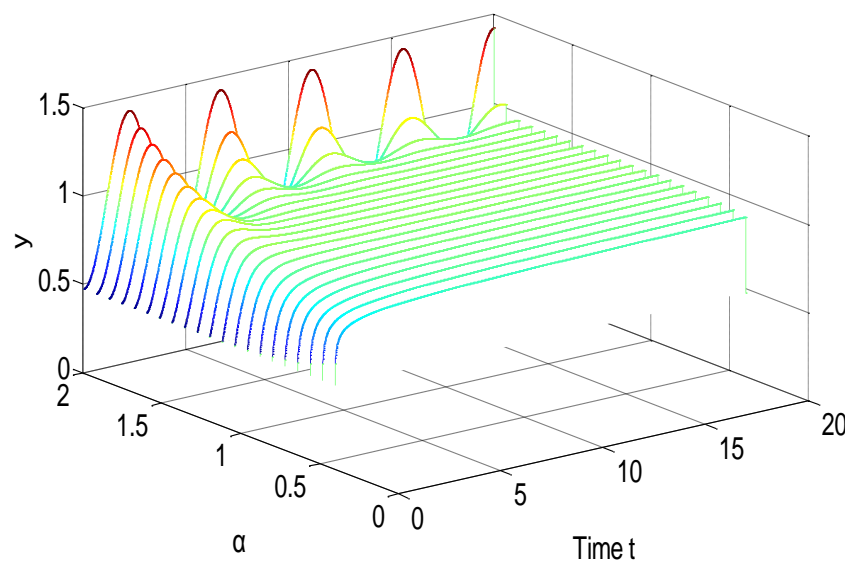

(c) The guide vane opening $y$

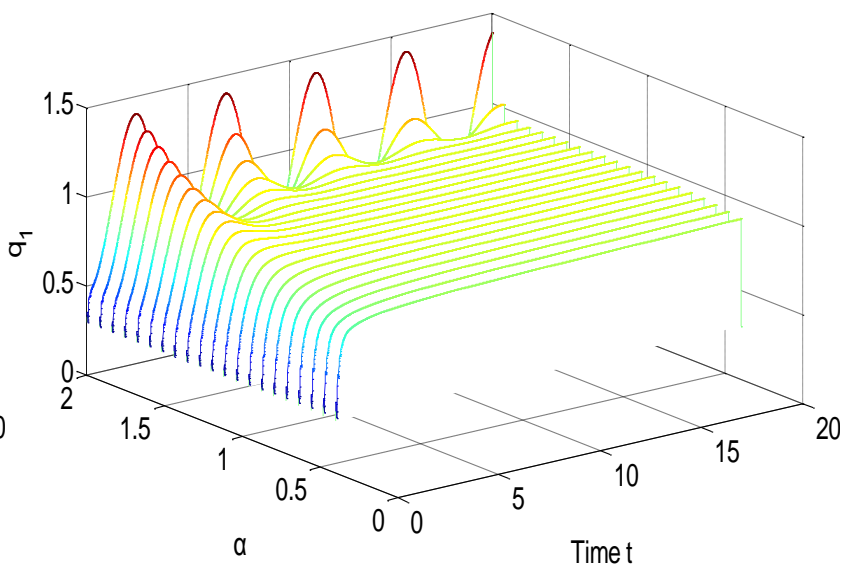

(b) The hydro-turbine discharge $q_{1}$

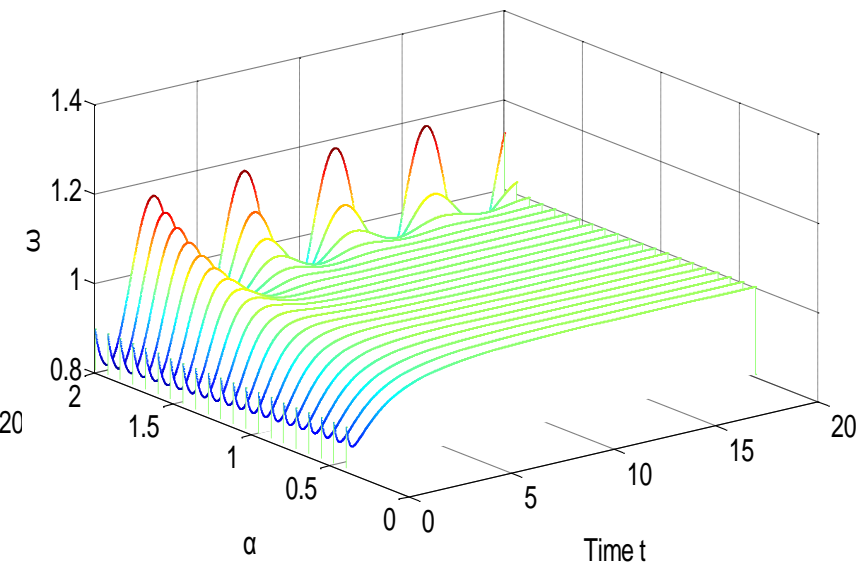

(d) The generator speed $\omega$

Fig. 8. The dynamic behaviors of the main four variables (the output power $P_{\mathrm{m}}$, the hydro-turbine discharge $q_{1}$, the guide vane opening $y$, the generator speed $\omega$ ) of hydro-turbine governing system hen $\alpha$ changes from 0.4 to 2 with the incremental steps of 0.08 .

From Fig. 8, we can get the change laws of the above four dynamic variables. The change laws of the overshoots for the above four variables are shown in Table 4. The change laws of the stable ranges of the four variables with the fractional order $\alpha$ varying are presented in Table 5. In addition, as $\alpha$ is more than around 2, the system is out of control (see Fig. 8).

Table 4 The change laws of the overshoot for the four variables (the output power $P_{\mathrm{m}}$, the hydro-turbine discharge $q_{1}$, the guide vane opening $y$, the generator speed $\omega$ ) of the hydro-turbine governing system with fractional item and time-lag. 


\begin{tabular}{ccccc}
\hline Variable & Ranges & Laws & Ranges & Laws \\
\hline$P_{m}$ & $(0.40,0.88)$ & $P_{m}=0.357(\alpha-0.4)+0.5387$ & $(0.88,2.00)$ & $P_{m}=1.27(\alpha-0.88)+0.7099$ \\
$q_{1}$ & $(0.40,1.04)$ & $q_{1}=0.07(\alpha-0.4)+0.9027$ & $(1.04,2.00)$ & $q_{1}=0.471(\alpha-1.04)+0.9479$ \\
$y$ & $(0.40,1.04)$ & $y=0.072(\alpha-0.4)+0.9045$ & $(1.04,2.00)$ & $y=0.494(\alpha-1.04)+0.9504$ \\
$\omega$ & $(0.40,1.04)$ & $\omega=1.004$ & $(1.04,2.00)$ & $\omega=0.1677(\alpha-1.04)+1.004$ \\
\hline
\end{tabular}

Table 5 The stable ranges of the four variables (the output power $P_{\mathrm{m}}$, the hydro-turbine discharge $q_{1}$, the guide vane opening $y$, the generator speed $\left.\omega\right)$ as the fractional order $\alpha$ is varying from 0.4 to 2.0 .

\begin{tabular}{ccccc}
\hline Variable & Ranges & Laws & Ranges & Laws \\
\hline$P_{m}$ & $(0.4,1.0)$ & $P_{m}=0.308(\alpha-0.4)+0.539$ & $(1.0,1.84)$ & $P_{m}=0.724$ \\
& $(1.84,1.92)$ & $P_{m}=2.538(\alpha-1.84)+0.724$ & $(1.92,2.0)$ & $P_{m}=14.51(\alpha-1.92)+0.927$ \\
$q_{1}$ & $(0.4,1.0)$ & $q_{1}=0.075(\alpha-0.4)+0.903$ & $(1.0,1.84)$ & $q_{1}=0.948$ \\
& $(1.84,1.92)$ & $q_{1}=0.913(\alpha-1.84)+0.948$ & $(1.92,2.0)$ & $q_{1}=4.675(\alpha-1.92)+1.021$ \\
$y$ & $(0.4,1.0)$ & $y=0.075(\alpha-0.4)+0.905$ & $(1,1.76)$ & $y=0.95$ \\
& $(1.76,1.92)$ & $y=0.44(\alpha-1.76)+0.95$ & $(1.92,2)$ & $y=5.05(\alpha-1.92)+1.021$ \\
$\omega$ & $(0.4,1.84)$ & $\omega=1.00$ & $(1.84,1.92)$ & $\omega=0.35(\alpha-1.84)+1$ \\
& $(1.92,2.0)$ & $\omega=1.22(\alpha-1.92)+1.028$ & & \\
\hline
\end{tabular}

\section{Conclusions}

In this paper, a fractional mathematical model with time-lag of a hydro-turbine governing system is proposed. Such model has been applied to the theory framework of the generalized Hamiltonian system. The Hamiltonian function, which is derived from the interval function, results into a reverse regulation function that allows to adequately describe the energy change of the hydro-turbine. Furthermore, some numerical experiments are presented to explore the effect of fractional order and time-lag on the characteristics of system variables. some key values of fractional order and time-lag where the state of the hydro-turbine governing system changes are highlighted. Finally, the difference between the function $y\left(t_{f}\right)$ which is solved from the fractional function $D^{\alpha} y\left(t_{1}\right)$ and the function $y\left(t_{d}-\tau\right)$ in nature has been discussed.

\section{Acknowledgements}

This work was supported by the scientific research foundation of National Natural Science 
Foundation-Outstanding Youth Foundation (51622906), National Science Foundation (51479173),

Fundamental Research Funds for the Central Universities (201304030577), Scientific research funds of Northwest A\&F University (2013BSJJ095), the scientific research foundation on water engineering of Shaanxi Province (2013slkj-12), the Science Fund for Excellent Young Scholars from Northwest A\&F University and Shaanxi Nova program (2016KJXX-55).

\section{References}

[1] A. Alidai, I. W. M. Pothof, Hydraulic performance of siphonic turbine in low head sites, Renew. Energy 75 (2015) 505-511.

[2] P. Duquesne, G. D. Ciocan, V. Aeschlimann, A. Bombenger, C. Deschenes, Pressure probe with five embedded flush-mounted sensors: unsteady pressure and velocity measurements in hydraulic turbine model, Exp. Fluids 54 (2013) DOI: 10.1007/s00348-012-1425-y.

[3] B. Strah, O. Kuljaca, Z. Vukic, Speed and active power control of hydro turbine unit, IEEE T. Energy Conver. 20 (2005) 424-434.

[4] K. Nagode, I. Skrjanc, Modelling and internal fuzzy model power control of a francis water turbine, Energies 7 (2014) 874-889.

[5] J. Y. Li, Q. J. Chen, Nonlinear dynamical analysis of hydraulic turbine governing systems with nonelastic water hammer effect, J. Appl. Math. (2014) DOI: 10.1155/2014/412578.

[6] X. L. Liu, C. Liu, Eigenanalysis of oscillatory instability of a hydropower plant including water conduit dynamics, IEEE T. Power Syst. 22 (2007) 675-681.

[7] Y. Zeng, L. X. Zhang, Y. K. Guo, K. H. Dong, Hydraulic decoupling and nonlinear hydro turbine model with sharing common conduit, Proceeding of the CSEE 32 (2012) 103-108. (in Chinese)

[8] Z. Y. Shen, Hydraulic turbine regulation, Beijing, China Water Power Press [in Chinese] (1998).

[9] Y. H. Zeng, W. X. Huai, Application of artificial neural network to predict the friction factor of open channel flow, Commun. Nonlinear Sci. 14 (2009) DOI: 10.1016/j.cnsns.2008.06.020.

[10] B. B. Xu, D. Y. Chen, H. Zhang, F. F. Wang, Modeling and stability analysis of a fractional-order Fracis hydro-turbine governing system. Chaos Solitons \& Fract. 75 (2015) 50-61.

[11] D. Y. Chen, C. Ding, X. Y. Ma, P. Yuan, D. D. Ba, Nonlinear dynamical analysis of hydro-turbine governing system with a surge tank, Appl. Math. Model 37 (2013) 14-15.

[12] H. Zhang, D. Y. Chen, B. B. Xu, F. F. Wang, Nonlinear modeling and dynamic analysis of hydro-turbine governing system in the process of load rejection transient, Energ. Convers. Manage. 90 (2015) 128-137.

[13] M. D. Ortigueira, J. A. T. Machado, What is a fractional derivative, J. Comput. phys. 293 (2015) 4-13.

[14] A. E. Matouk, A. A. Elsadany, E. Ahmed, H. N. Agiza, Dynamical behavior of fractional-order 
Hastings-Powell food chain model and its discretization, Commun. Nonlinear Sci. 27 (2015) 153-167.

[15] D. Baleanu, R. L. Magin, S. Bhalekar, V. Daftardar-Gejji, Chaos in the fractional order Bloch equation with delay, Commun. Nonlinear Sci. 25 (2015) 41-49.

[16] R. H. Wagoner, M. Li, Simulation of springback: Through-thickness integration, Int. J. Plasticity 23 (2007) 345-360.

[17] R. Metzler, T. F. Nonnenmacher, Fractional relaxation processes and fractional rheological models for the description of a class of viscoelastic materials, Int. J. Plasticity 19 (2003) 941-959.

[18] C. A. K. Kwuimy, G. Litak, C. Nataraj, Nonlinear analysis of energy harvesting systems with fractional order physical properties, Nonlinear dynam. 80 (2015) 491-501.

[19] T. Sardar, S. Rana, J. Chattopadhyay, A mathematical model of dengue transmission with memory, Commun. Nonlinear Sci. 22 (2015) 511-525.

[20] J. B. Hu, G. P. Lu, S. B. Zhang, L. D. Zhao, Lyapunov stability theorem about fractional system without and with delay, Commun. Nonlinear Sci. 20 (2015) DOI: 10.1016/j.cnsns.2014.05.013.

[21] F. Armero, J. C. Simo, Apriori stability estimates and unconditionally stable product formla algorithms for nonlinear coupled thermoplasticity, Int. J. Plasticity 9 (1993) 749-782.

[22] J. A. T. Machado, A. Galhano, Fractional dynamics: a statistical perspective, J. Comput. nonlin. Dyn. 3 (2008) DOI: 10.1115/1.2833481.

[23] Y. Zeng, L. X. Zhang, T. M. Xu, Y. K. Guo, Hamiltonian model of nonlinear hydraulic turbine with elastic water column, Proceeding of the CSEE 28 (2010) 525-520. (in Chinese)

[24] D. Z. Cheng, Z. R. Xi, Q. Lu, S. W. Mei, Geometric structure of generalized controlled Hamiltonian systems and its application, Science in China Series E-Technological Sciences 43 (2000) 365-379.

[25] G. Escobar, A. J. Vander Schaft, R. Ortega, A Hamiltonian viewpoint in the modeling of switching power converters, Automatica 35 (1999) 445-452.

[26] S. M. Yu, Y. Q. Yao, S. F. Shen, W. X. Ma, Bi-integrable couplings of a Kaup-Newell type soliton hierarchy and their bi-Hamiltonian, Commun. Nonlinear Sci. 23 (2015) 366-377.

[27] F. Sugino, T. Yoneya, Stochastic Hamiltonians for noncritical string field theories from double-scaled matrix models, Phys. Rev. D 53 (1996) 4448-4488.

[28] X. G. Geng, R. M. Li, B. Xue, A new hierarchy of integrable equation with peakons and cuspons and its bi-Hamiltonian structure, Appl. Math. Lett. 46 (2015) 64-69.

[29] Y. Z. Wang, D. Z. Cheng, S. S. Ge, Approximate dissipative Hamiltonian realization and construction of local Lypaunov functions, Syst. Control Lett. 56 (2007) 141-149.

[30] N. Klemen, S. Igor, Modelling and internal fuzzy model power control of a francis water turbine, energies 7 (2014) 874-889.

[31] IEEE Working Group: Hydraulic-turbine and turbine control-models for system dynamic studies, IEEE T. Power Syst. 7 (1992) 167-79.

[32] Y. Z. Wang, D. Z. Cheng, X. M. Hu, Problems on time-varying port-controlled Hamiltonian system: geometric structure and dissipative realization - Brief paper, Automatica 41 (2005) 717-723.

[33] Y. Zeng, L. X. Zhang, Y. K. Guo, J. Qian, C. L. Zhang, The generalized Hamiltonian model for the shafting transient analysis of the hydro turbine generating sets, Nonlinear Dynam. 76 (2014) 1921-1933.

[34] Y. Zeng, L. X. Zhang, J. Qian, T. M. Xu, Y. K. Guo, Differential algebra model simulation for hydro turbine with elastic water column, Journal of drainage and irrigation machinery engineering 32(8) (2014) 691-697. (in Chinese) 